\title{
Evaluation of peripapillary choroidal thickness in patients with normal-tension glaucoma
}

\author{
Kazuyuki Hirooka ${ }^{*}$, Kaori Tenkumo $^{\dagger}$, Atsushi Fujiwara ${ }^{\dagger}$, Tetsuya Baba ${ }^{\dagger}$, Shino Sato $^{\dagger}$ and Fumio Shiraga ${ }^{\dagger}$
}

\begin{abstract}
Background: To compare peripapillary choroidal thickness measurements between normal and normal-tension glaucoma eyes.

Methods: Cross-sectional comparative study. 50 normal and 52 normal-tension glaucoma subjects were enrolled in the study. Peripapillary choroidal thickness was measured with spectral-domain optical coherence tomography and enhanced depth imaging. After obtaining circular B-scans around the disc, choroidal thicknesses were calculated based on the exported segmentation values. Visual fields were measured using automated perimetry. Difference in peripapillary choroidal thickness between the normal subjects and the patients with normal-tension glaucoma was analyzed.
\end{abstract}

Results: There were no significant differences in age, axial length, or refraction between the two groups. Peripapillary choroidal thickness was inversely correlated with age in both the normal $(r=-0.287, P=0.04)$ and normal and normal-tension glaucoma $(r=-0.322, P=0.02)$ groups. Peripapillary choroidal thickness of inferonasal (125 vs $148 \mu \mathrm{m}, P<0.05$ ), inferior (101 vs $122 \mu \mathrm{m}, P<0.05$ ), or inferotemporal (100 vs $127 \mu \mathrm{m}, P<0.05$ ) regions were significantly thinner in the normal-tension glaucoma group as compared to normal subjects. Superior visual hemifield defect was significantly worse than inferior visual hemifield defect in normal and normal-tension glaucoma patients.

Conclusion: As compared to normal subjects, peripapillary choroidal thickness was significantly thinner in the normal and normal-tension glaucoma patients, at least in some locations.

Keywords: Peripapillary choroidal thickness, Enhanced depth imaging optical coherence tomography,

Normal-tension glaucoma

\section{Background}

Normal-tension glaucoma (NTG) accounts for 92\% of primary open-angle glaucoma (POAG) in Japanese patients [1]. This clinical term is often used to describe patients with open-angle glaucoma (OAG) in whom the measured untreated intraocular pressure (IOP) is always within a statistically normal range. Multicenter clinical trials have confirmed the value of reducing the IOP in POAG $[2,3]$ and NTG $[4,5]$ patients. There has yet to be a consensus regarding the specific relationship between the IOP and NTG. However, elevated IOPs are not always associated with glaucomatous optic neuropathy

\footnotetext{
*Correspondence: kazuyk@med.kagawa-u.ac.jp

${ }^{\dagger}$ Equal contributors

Department of Ophthalmology, Kagawa University Faculty of Medicine, 1750-1 Ikenobe, Miki, Kagawa 761-0793, Japan
}

(GON), and in many cases, a progression of the GON has been observed after lowering the IOP. Thus, these findings suggest that in addition to the presence of an elevated IOP, there may be other factors that play an important role in glaucoma pathogenesis. After the original anatomical findings of peripapillary chorioretinal atrophy were first reported [6], many authors speculated that the ciliary circulation, particularly the choroidal supply to the papillary area, could be an etiological factor in glaucoma $[7,8]$.

Abnormal choroidal blood supply has been suggested to be one factor responsible for the occurrence of GON in OAG patients [7]. Yin et al. [9] found that POAG eyes had the thinnest choroid both globally and in the peripapillary region. Recently, there has been an increasing interest in determining the specific role that the choroid

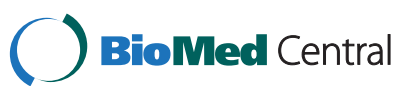


plays in the pathogenesis of OAG. In general, there is a depth-dependent roll-off in the sensitivity of the spectral-domain optical coherence tomography (OCT) instruments $[10,11]$. Due to these potential problems, this led to the development of enhanced depth imaging (EDI) spectral-domain OCT, which subsequently made it possible to perform in vivo cross-sectional imaging of the choroid [10]. Other investigators have reported that the measurement of choroidal thickness with the use of OCT a longer wavelength $[12,13]$. Through use of this new methodology, investigators have been able to examine the choroidal thickness in glaucoma patients [11-15]. While some of these investigations have reported finding that the choroidal thickness did not seem to differ between normal and glaucoma patients [14-17], Usui et al. [17] have reported that the choroidal thickness in highly myopic NTG was significantly thinner as compared to controls. However, this latter study only included spherical equivalent refractive errors between -6 and -12 diopters and axial lengths that exceeded $26.5 \mathrm{~mm}$.

We recently showed that the choroidal thickness at $3 \mathrm{~mm}$ nasal from the fovea was significantly thinner in NTG eyes as compared to normal subjects and also correlated with the mean deviation slope [18]. However, choroidal thickness was measured in only one peripapillary location. The purpose of the current study was to determine if there were differences in the EDI OCT peripapillary choroidal thickness measurements obtained in normal and NTG eyes.

\section{Methods}

Consecutive NTG patients who visited the Kagawa University Hospital, Kagawa, Japan between May 2011 and August 2011 were recruited for the study. One eye per subject was randomly selected for the study. Inclusion criteria included a cylinder within $\pm 2.0 \mathrm{D}$. In order to be enrolled, subjects were required to have had no previous ocular surgery. Subjects were excluded if they had any history of retinal diseases (e.g., diabetic retinopathy, macular degeneration, retinal detachment) or laser therapy, had a poor image quality because of unstable fixation, or had severe cataract. Exclusion criteria also included any history of treatment with medications that could affect retinal thickness, such as intravitreal antiVEGF therapy. The same examiner performed the EDI OCT examinations in all cases in the morning. All subjects underwent visual acuity, refraction, central and peripheral fields, slit lamp, and gonioscopy examinations. All NTG patients had typical glaucomatous optic disc damage with nerve fiber bundle defects and open angle. NTG was diagnosed when there was an untreated peak IOP of $\leq 21 \mathrm{mmHg}$, which included the 24-hour fluctuation measurements. Glaucomatous eyes were defined as eyes exhibiting structural glaucomatous changes (vertical cup-disc asymmetry between fellow eyes of $\geq 0.2$, a cupto-disc ratio of $\geq 0.6$, and neuroretinal rim narrowing, notches, localized pallor, or retinal nerve fiber layer defects with glaucomatous visual field (VF) loss in the corresponding hemifield). Glaucomatous VF defects were defined as defects with consecutive, repeated abnormal standard automated perimetry results ( $\geq 2$ contiguous points with a sensitivity loss of $P<0.05$ in the superior or inferior arcuate areas, a $10 \mathrm{~dB}$ difference across the nasal horizontal midline at $>2$ adjacent locations, or an abnormal glaucoma hemifield test result). Other inclusion criteria for the normal subjects included an IOP $\leq 21 \mathrm{mmHg}$ and a normal ophthalmoscopic appearance of the optic nerve. All eligible subjects received a detailed explanation about the study and signed an informed consent form in accordance with the principles embodied in the Declaration of Helsinki. This study was approved by the institutional review board at the Kagawa University Hospital.

The examination protocol was conducted in a seated, resting position and included measurements of the axial length and keratometry in both eyes using an IOLMaster (Carl Zeiss Meditec, Dublin, CA). Subsequently spectral domain OCT (SD-OCT) scans of the peripapillary regions were performed using the Heidelberg Spectralis (Heidelberg Engineering, Heidelberg, Germany). The SD-OCT images were obtained by using enhanced depth imaging. With this method, the focus is manually placed more posteriorly than would normally be done during standard retinal SD-OCT imaging, as this improves the resolution of the choroidal detail. However, because of this, the images that are produced are inverted. The peripapillary region was scanned using a $360^{\circ}, 3.4 \mathrm{~mm}$ diameter circle scan that was centered on the optic disc (Figure 1A). A reader who was masked to the clinical patient data used the provided Heidelberg Eye Explorer software (version 1.5.12.0; Heidelberg Engineering) to manually delineated the choroidal thickness. This thickness was defined as the area of visible choroidal vasculature between the outer retinal pigment epithelial border and the inner scleral wall (Figure 1B). Subsequently, the Humphrey central 30-2 full-threshold program (C30-2 program) was used to determine the visual field, which was defined as reliable when fixation losses, falsepositive errors, and false-negative errors were less than $20 \%$.

Statistical analysis was performed using SPSS for Windows (SPSS Inc., Chicago, IL). Differences for age, IOP, axial length, refraction and visual hemifield defects were analyzed using an independent Student's $t$-test. The Shapiro-Wilk test was used to determine the normality of the distribution of the choroidal thickness data. The choice of the Mann-Whitney $U$ or independent Student's $t$-test to study the significance of difference was 


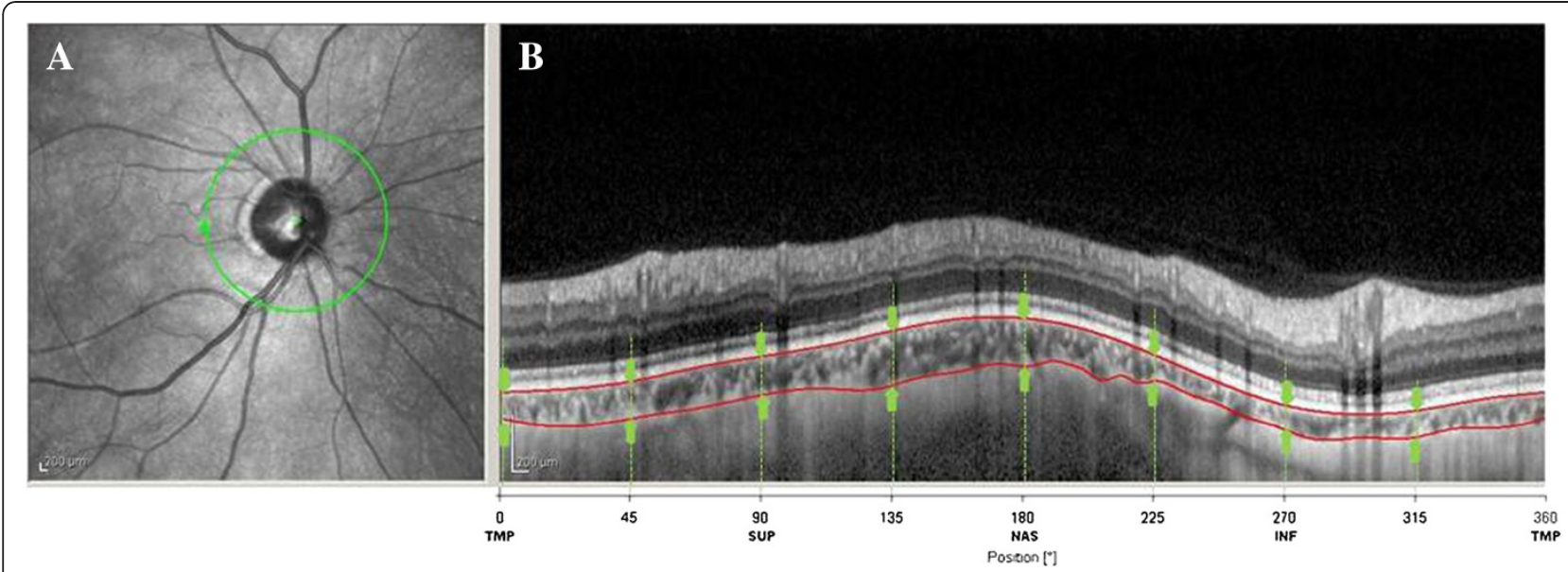

Figure 1 The location of the scan used (A) and images from the $360^{\circ}$ peripapillary EDI OCT scans (B). Peripapillary choroidal thickness (between up-pointing arrow and down-pointing arrow) was measured from the outer border of the retinal pigment epithelium (RPE) to the inner border of the sclera (arrowheads).

based on the presence or absence of the normality of distribution. Gender differences were analyzed by Fisher's exact probability test. The correlation between the axial length, age, refraction, retinal nerve fiber layer (RNFL) thickness or mean deviation (MD) and the choroidal thickness was analyzed using a Spearman's rank correlation coefficient test. A $P$ value less than 0.05 was considered statistically significant. The data are presented as mean \pm standard deviation (SD).

\section{Results}

A total of 103 subjects (50 normal subjects and 52 NTG patients) met the eligibility criteria. There were no significant differences noted between normal and NTG groups for age (62.4 vs 66.2 years, $P=0.10)$, gender $(P=$ 0.12 ), axial length (24.4 vs $24.9 \mathrm{~mm}, P=0.10)$, refraction $(-2.2$ vs -2.6 diopter, $P=0.57)$, or corneal refraction (43.8 vs 43.4 diopter, $P=0.15$ ) (Table 1 ). However, the IOP in the NTG group was significantly lower than that

Table 1 Clinical characteristics of the study participants

\begin{tabular}{lccc}
\hline & Normal & NTG & P value \\
\hline $\mathrm{N}$ & 50 & 52 & \\
Age $(\mathrm{y})$ & $62.4 \pm 10.0$ & $66.2 \pm 13.1$ & 0.10 \\
Gender (M/F) & $28 / 22$ & $21 / 31$ & 0.12 \\
Axial length (mm) & $24.4 \pm 1.4$ & $24.9 \pm 1.5$ & 0.10 \\
IOP (mmHg) & $14.1 \pm 2.8$ & $12.8 \pm 1.9$ & 0.004 \\
MD (dB) & & $-12.1 \pm 7.3$ & \\
Refraction (D) & $-2.2 \pm 4.0$ & $-2.6 \pm 3.7$ & 0.57 \\
Corneal refraction (D) & $43.8 \pm 1.1$ & $43.4 \pm 1.3$ & 0.15 \\
\hline
\end{tabular}

NTG, normal-tension glaucoma; $M$, male; $F$, female; $I O P$, intraocular pressure; $M D$, mean deviation; $D$, diopter. observed in the normal subjects (14.1 vs $12.8 \mathrm{mmHg}$, $P=0.004)$.

Mean peripapillary choroidal thicknesses were $148.8 \pm$ $53.3 \mu \mathrm{m}$ and $128.1 \pm 44.6 \mu \mathrm{m}$ in the normal and NTG subjects, respectively $(P=0.04)$ (Table 2$)$. Peripapillary choroidal thicknesses of the inferonasal $(P=0.04)$, inferior $(P=0.03)$, or inferotemporal $(P=0.01)$ areas in the NTG group were significantly thinner than those observed in the normal subjects (Table 2). In normal subjects, age was correlated with the superonasal $(\mathrm{r}=$ -0.322, $P=0.02)$, nasal $(\mathrm{r}=-0.415, P=.003)$, inferonasal $(\mathrm{r}=-0.347, P=0.01)$, inferior $(\mathrm{r}=-0.291, P=0.04)$ and average $(\mathrm{r}=-0.287, P=0.04)$ (Figure $2 \mathrm{~A}$ ) choroidal thicknesses. In NTG subjects, age was correlated with superior $(\mathrm{r}=-0.307, P=0.03)$, superonasal $(\mathrm{r}=-0.339$, $P=0.01)$, nasal $(\mathrm{r}=-0.402, P=0.003)$, inferonasal $(\mathrm{r}=$ -0.371, $P=0.006)$, inferior $(\mathrm{r}=-0.271, P=0.049)$ and average $(\mathrm{r}=-0.322, P=0.02)$ (Figure $2 \mathrm{~B})$ choroidal

Table 2 Choroidal thickness in normal and NTG eyes

\begin{tabular}{lccc}
\hline Location & \multicolumn{2}{c}{ Choroidal thickness $(\boldsymbol{\mu m})$} & \multirow{2}{*}{ P value } \\
\cline { 2 - 3 } & Normal & NTG & \\
\hline Temporal & $142.6 \pm 69.1$ & $129.3 \pm 55.9$ & 0.29 \\
Superotemporal & $149.6 \pm 62.2$ & $131.5 \pm 51.1$ & 0.11 \\
Superior & $163.0 \pm 56.1$ & $143.1 \pm 51.5$ & 0.07 \\
Superonasal & $169.8 \pm 57.5$ & $148.5 \pm 53.5$ & 0.06 \\
Nasal & $168.5 \pm 59.4$ & $146.4 \pm 55.1$ & 0.05 \\
Inferonasal & $148.2 \pm 60.3$ & $125.0 \pm 49.3$ & 0.04 \\
Inferior & $121.8 \pm 55.5$ & $100.8 \pm 37.0$ & 0.03 \\
Inferotemporal & $127.1 \pm 60.7$ & $100.0 \pm 42.7$ & 0.01 \\
Average & $148.8 \pm 53.3$ & $128.1 \pm 44.6$ & 0.04 \\
\hline
\end{tabular}

NTG, normal-tension glaucoma. 

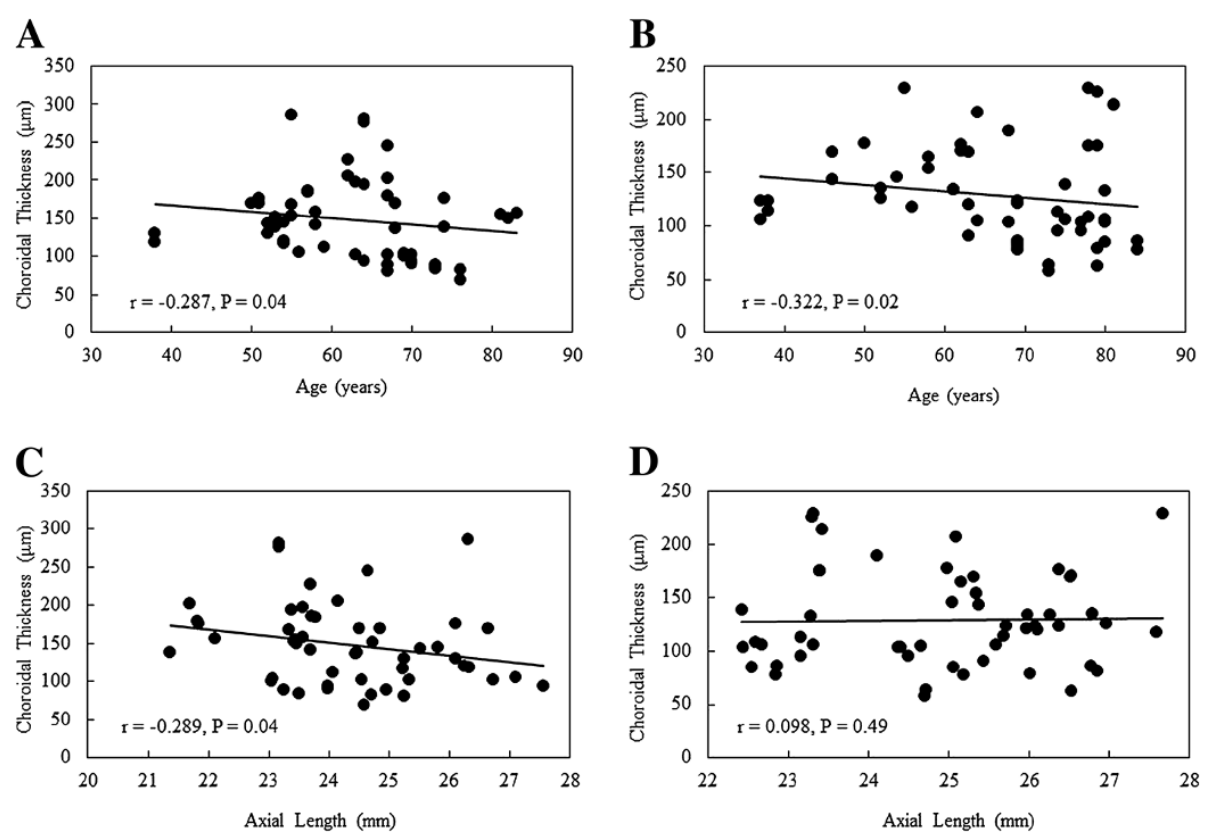

Figure 2 Scatter plot of the simple linear regression analysis between the age and (A) the mean peripapillary choroidal thickness in normal subjects, and (B) the mean peripapillary choroidal thickness in patients with normal-tension glaucoma (NTG). Scatter plot of the simple linear regression analysis between the axial length and $(\mathbf{C})$ the mean peripapillary choroidal thickness in normal subjects, and (D) the mean peripapillary choroidal thickness in patients with NTG.

thicknesses. In the normal subjects, axial length was correlated with temporal $(\mathrm{r}=-0.438, P=0.001)$, superotemporal $(\mathrm{r}=-0.346, P=0.01)$, inferotemporal $(\mathrm{r}=$ $-0.334, P=0.02)$ and average $(r=-0.289, P=0.04)$ (Figure 2C) choroidal thicknesses. In the normal subjects, the refraction was only correlated with the temporal choroidal thickness $(r=0.346, P=0.01)$. In the NTG subjects, none of the measurements were correlated with either the axial length (Figure 2D) or refraction (all $P>0.05$ ). There was also no correlation noted between the IOP and the choroidal thickness (all $P>0.05)$. Spearman's rank correlation coefficient test

Table 3 Sperman's rank correlation between RNFL thickness and choroidal thickness by peripapillary location

\begin{tabular}{lcc}
\hline Location & Sperman's rank & $P$ value \\
\cline { 2 - 2 } & correlation coefficient $(\mathbf{r})$ & \\
\hline Temporal & -0.076 & 0.63 \\
Superotemporal & 0.037 & 0.81 \\
Superior & 0.358 & 0.02 \\
Superonasal & 0.011 & 0.95 \\
Nasal & 0.085 & 0.59 \\
Inferonasal & -0.191 & 0.21 \\
Inferior & -0.052 & 0.74 \\
Inferotemporal & 0.102 & 0.47 \\
\hline
\end{tabular}

$R N F L$, retinal nerve fiber layer demonstrated that RNFL thickness and peripapillary choroidal thickness measurements were not significantly correlated for any peripapillary location $(\mathrm{r}<0.102$, $P>0.21)$, except superior $(\mathrm{r}=0.358, P=0.02)$ (Table 3 ). There was no significantly correlation between MD and mean peripapillary choroidal thickness $(r=0.188$, $P=0.18)$.

Mean total deviation of the inferior visual hemifield was $-10.4 \pm 8.0 \mathrm{~dB}$, while mean total deviation of the superior visual hemifield was $-12.9 \pm 8.1 \mathrm{~dB}$ (Table 4). There was a significant difference between the inferior and superior visual hemifield defects $(P=0.04)$.

\section{Discussion}

When compared to the eyes of the normal subject, the NTG eyes had a significantly thinner mean peripapillary choroidal thickness. Although both age and axial length were correlated with the mean peripapillary choroidal thickness in the normal subjects, only age was correlated with the mean peripapillary choroidal thickness in the NTG patients. The inferior peripapillary choroidal thickness was also thinner in the NTG patients as compared to the control subjects. Interestingly, the superior visual

Table 4 Visual hemifield defects in the NTG patients

\begin{tabular}{lcc}
\hline Superior & Inferior & P value \\
\hline$-12.9 \pm 8.1 \mathrm{~dB}$ & $-10.4 \pm 8.0 \mathrm{~dB}$ & 0.04 \\
\hline NTG, normal-tension glaucoma. & &
\end{tabular}


hemifield defect was worse than the inferior visual hemifield defect in the NTG patients.

Previous studies that performed histological analyses of glaucomatous eyes have reported finding both increases and decreases in the choroidal thicknesses $[12,19,20]$. However, since fixation of the choroid before histologic analysis causes shrinkage, this will affect the thickness measurements. As such, histological analysis can only provide a rough estimate of the in vivo choroid thickness. Mwanza et al. [16] recently examined EDI OCT measurements of the choroidal thickness and reported finding no differences between the normal, NTG, and POAG subjects. However, the macular choroidal thickness was only measured in 38 normal and 20 NTG subjects, which was smaller than our current study. Usui et al. [17] examined subjects using highpenetration OCT and showed that the choroidal thickness was significantly thinner in high myopic NTG patients as compared to high myopic controls. They concluded that this choroidal thinning was related to the highly myopic NTG.

Generalized choroidal thinning is associated with vessel loss, and occurs predominantly in the inner choroids [12]. It is possible that this phenomenon could reduce the blood flow volume in the low-pressure choroidal bed. Previous evidence has shown that the peripapillary choroid is responsible for supplying the prelaminar region, with the most convincing evidence for this derived from fluorescence angiographic studies [21,22]. However, there are other postmortem morphological studies that have reported evidence that strongly supports the theory that the peripapillary choroid does not supply the prelaminar region of the optic nerve head [23-26]. However, in the Cioffi \& Van Buskirk study, [26] they did find that some branches of the circle of Haller and Zinn (CHZ) and the short posterior ciliary arteries (PCAs) coursed through the choroid and ultimately supplied the prelaminar region. Hayreh [27] further discussed this discrepancy and pointed out that once the short and long PCAs and the branches of the $\mathrm{CHZ}$ had pierced the sclera, they then essentially became a part of the choroid and were no longer considered to be branches of the PCAs. Thus, the apparent confusion and conflicting speculations appear to be simply a matter of semantics.

There are potential limitations in our study. Although choroidal thickness seems to be influenced by diastolic blood pressure [15], there was no data on the blood pressure in the two groups of patients. Second, the current operating software of Heidelberg Spectralis OCT does not provide automatic segmentation of the choroid. However, Yamashita et al. [28] recently reported that intraobserver intraclass correlation coefficients (ICCs) for subfoveal choroidal thickness was $0.976(P<0.001)$. The measurements were extremely reproducible. Third, there seems to be significant diurnal variation of choroidal thickness $[29,30]$. The choroidal thickness decreased from 9:00 AM through the time point at 11:00 AM [30]. The OCT scans were performed in the morning in our study.

\section{Conclusion}

In conclusion, our current study demonstrated that the mean peripapillary choroidal thickness was significantly thinner in the NTG eyes when compared to normal subjects. In the NTG patients, the peripapillary choroidal thickness of the inferonasal, inferior, or inferotemporal regions decreased in conjunction with a corresponding worsening of the visual hemifield defect.

\section{Competing interests}

The authors declare that they have no competing interests.

\section{Authors' contributions}

$\mathrm{KH}$ Conception and design. AF Acquisition of data. KH Analysis and interpretation of data. KH, KT Preparation. KH, FS Review. KH, KT, AF, TB, SS, FS Approval of manuscript. All authors read and approved the final manuscript.

\section{Acknowledgement}

We are grateful to Forte Science Communications for proofreading of this manuscript.

Received: 25 April 2012 Accepted: 20 July 2012

Published: 28 July 2012

\section{References}

1. Iwase A, Suzuki Y, Araie M, et al: Tajimi Study Group, Japan Glaucoma Society. The prevalence of primary open-angle glaucoma in Japanese: the Tajimi Study. Ophthalmology 2004, 111(9):1641-1648.

2. The AGIS Investigators: The Advanced Glaucoma Intervention Study (AGIS):7, The relationship between control of intraocular pressure and visual field deterioration. Am J Ophthalmol 2000, 130(4):429-440.

3. Lichter PR, Musch DC, Gillespite BW, et al: CIGTS Study Group: Interim clinical outcomes in the Collaborative Initial Glaucoma Treatment Study comparing initial treatment randomized to medications or surgery. Ophthalmology 2001, 108(11):1943-1953.

4. Collaborative Normal-tension Study Group: The effectiveness of intraocular pressure reduction in the treatment of normal-tension glaucoma. Am J Ophthalmol 1998, 126(4):498-505.

5. Heijl A, Leske MC, Bengtsson B, Hyman L, Bengtsson B, Hussein M: Early Manifest Glaucoma Trial Group: Reduction of intraocular pressure and glaucoma progression: results from the Early Manifest Glaucoma Trial. Arch Ophthalmol 2002, 120(10):1268-1279.

6. Elsching A: Glaucoma. In Handbuch der speziellen pathologischen anatomie und histologievol II (Ague), part I. Edited by Henke F, Lubarsch O. Berlin: Springer; 1928:873.

7. Hayreh SS: Blood supply of the optic nerve head and its role in optic atrophy, glaucoma, and oedema of the optic disc. Br J Ophthalmol 1969, 53(11):721-748.

8. Hamard P, Hamard H, Dufaux J, Quesnot S: Optic nerve head blood flow using a laser Doppler velocimeter and haemorheology in primary open angle glaucoma and normal pressure glaucoma. Br J Ophthalmol 1994, 78(6):449-453

9. Yin ZQ, Vaegan, Millar TJ, Beaumont P, Sarks S: Widespread choroidal insufficiency in primary open-angle glaucoma. J Glaucoma 1997, 6(1):23-32.

10. Spaide RF, Koizumi H, Pozzoni MC: Enhanced depth imaging spectral-domain optical coherence tomography. Am J Ophthalmol 2008, 146(4):496-500.

11. Ho J, Branchini L, Regatieri C, et al: Analysis of normal peripapillary choroidal thickness via spectral domain optical coherence tomography. Ophthalmology 2011, 118:2001-2007. 
12. Esmaeelpour M, Povazay B, Hermann B, et al: Three-dimensional 1060-nm OCT: choroidal thickness maps in normal subjects and improved posterior segment visualization in cataract patients. Invest Ophthalmol Vis Sci 2010, 51(10):5260-5266.

13. Huber R, Adler DC, Srinivasan VJ, Fujimoto JG: Fourier domain mode locking at $1050 \mathrm{~nm}$ for ultra-high-speed optical coherence tomography of the human retina at 236,000 axial scans per second. Opt Lett 2007, 32(14):2049-2051.

14. Ehrlich JR, Peterson J, Parlitsis $G$, et al: Peripapillary choroidal thickness in glaucoma measured with optical coherence tomography. Exp Eye Res 2011, 92(3):189-194

15. Maul EA, Friedman DS, Chang DS, et al: Choroidal thickness measured by spectral domain optical coherence tomography: factors affecting thickness in glaucoma patients. Ophthalmology 2011, 118(3):1571-1579.

16. Mwanza JC, Hochberg JT, Banitt MR, Feuer WJ, Budenz DL: Lack of association between glaucoma and macular choroidal thickness measured with enhanced depth-imaging optical coherence tomography. Invest Ophthalmol Vis Sci 2011, 52(6):3430-3435.

17. Usui S, Ikuno Y, Miki A, et al: Evaluation of the choroidal thickness using highpenetration optical coherence tomography with long wavelength in high myopic normal-tension glaucoma. Am J Ophthalmol 2012, 153(1):10-16.

18. Hirooka K, Fujiwara A, Shiragami T, Baba T, Shiraga F: Relationship between progression of visual field damage and choroidal thickness in eyes with normal-tension glaucoma. Clin Exp Ophthalmol, in press.

19. Cristini G, Cennamo G, Daponte P: Choroidal thickness in primary glaucoma. Ophthalmologica 1991, 202(2):81-85.

20. Kubota T, Jonas JB, Naumann OH: Decreased choroidal thickness in eyes with secondary angle closure glaucoma. An aetiological factor for deep retinal changes in glaucoma? Br J Ophthalmol 1993, 77(7):430-432.

21. Hayreh SS: Anterior ischemic optic neuropathy,I. Terminology and pathogenesis. Br J Ophthalmol 1974, 58(12):955-963.

22. Hayrh SS: Inter-individual variation in blood supply of the optic nerve head. Its importance in various ischemic disorders of the optic nerve head, and glaucoma, low-tension glaucoma and allied disorders. Doc Ophthalmol 1985, 59(3):217-246.

23. Lieberman MF, Maumenee AE, Green WR: Histologic studies of the vasculature of the anterior optic nerve. Am J Ophthlmol 1976, 82(3):405-423.

24. Zhao Y, Li FM: Microangioarchitecture of optic papilla. Jpn J Ophthalmol 1987, 31(1):147-159.

25. Cioffi GA, Van Buskirk EM: Vasculature of the anterior optic nerve and peripapillary choroid. In The Glaucomas. 2nd edition. St. Louis: Mosby; 1996:177-188.

26. Onda E, Cioffi GA, Bacon DR, Van Buskirk EM: Microvasculature of the human optic nerve. Am J Ophthalmol 1995, 120(1):92-102.

27. Hayreh SS: The blood supply of the optic nerve head and the evaluation of it-myth and reality. Prog Retin Eye Res 2001, 20(5):563-593.

28. Yamashita T, Yamashita T, Shirasawa M, et al: Repeatability and reproducibility of subfoveal choroidal thickness in normal eye of Japanese using SD-OCT devices. Invest Ophthalmol Vis Sci 2012, 53(3):1102-1107.

29. Brown JS, Flitcroft DI, Ying GS, et al: In vivo human choroidal thickness measurements: evidence for diurnal fluctuations. Invest Ophthalmol Vis Sci 2009, 50(1):5-12

30. Tan CS, Ouyang Y, Ruiz H, Sadda SR: Diurnal variation of choroidal thickness in normal, healthy subjects measured by spectral domain optical coherence tomography. Invest Ophthalmol Vis Sci 2012, 53(1):261-266.

doi:10.1186/1471-2415-12-29

Cite this article as: Hirooka et al.: Evaluation of peripapillary choroidal

thickness in patients with normal-tension glaucoma. BMC Ophthalmology 2012 12:29

\section{Submit your next manuscript to BioMed Central and take full advantage of:}

- Convenient online submission

- Thorough peer review

- No space constraints or color figure charges

- Immediate publication on acceptance

- Inclusion in PubMed, CAS, Scopus and Google Scholar

- Research which is freely available for redistribution 